\title{
APROXIMACIONES A LOS PRINCIPIOS DE LA REFORMA PROCESAL CIVIL
}

\author{
APPROACHES TO THE PRINCIPLES OF THE CIVIL PROCEEDINGS REFORM
}

JoRDI D ELGADO CASTRO ${ }^{1}$

\begin{abstract}
Resumen:
El presente comentario pretende relevar los principios de la reforma procesal civil. Además, de forma especial se adentra en señalar algunas ventajas del proceso escrito rebajando la euforia existente entorno a los beneficios aparejados del principio de la oralidad.
\end{abstract}

Palabras clave: Reforma Procesal Civil - Principios Básicos - Oralidad

\begin{abstract}
The purpose of the present commentary is to highlight the principles of the civil proceedings reform. In addition, special attention is given to expounding some of the advantages of written proceedings to reduce the current euphoria about the benefits related to the principle of oral procedures.
\end{abstract}

Key words: Civil Proceedings Reform - Basic Principles - Oral procedures

1 Abogado del Ilustre Colegio de Abogados de Barcelona. Doctor en Derecho, Universidad de Barcelona, Académico de la Universidad Católica de Temuco. Director de la Revista Chilena de Derecho y Ciencia Política. Correo: jdelgado@uct.cl 


\section{Planteamiento}

Uno de los hitos más importantes en el movimiento de las llamadas reformas procesales es, sin duda, la reforma procesal civil. No solamente porque a los procesalistas nos vayan a dar qué opinar para un buen rato, sino porque es el procedimiento más importante, común a los demás y quizá va a conseguir que entendamos -de nuevo- que el derecho de familia es derecho civil. Pero, sin duda, la génesis de este movimiento comenzó con la reforma procesal penal que sentó las bases de un nuevo sistema con predominio de la oralidad al que fueron siguiendo las demás reformas ${ }^{2}$.

Desde un punto de vista más práctico y teniendo en cuenta que el procedimiento es el aspecto exterior de la actividad jurisdiccional ${ }^{3}$ también va a resultar muy interesante. El ciudadano que es quien sustenta nuestro sistema de justicia va a poder observar una infraestructura ${ }^{4}$ más adecuada a sus necesidades y una respuesta judicial que, ojalá, guarde la nota de efectiva al ser entregada con mayor brevedad.

Al diseñar un sistema procesal podemos adoptar dos posturas: En primer lugar, podemos configurar un sistema de libertad procesal (como el establecido en el Decreto de 3 de Brumario del año 2 - Es decir en 1793 en la Revolución Francesa) en que se minimicen al máximo las formas e incluso desaparezca en esa especie de caos la profesión del abogado. O bien, en segundo término, se puede optar por un sistema de legalidad en las formas procesales en que prácticamente todo está regulado y, que en ocasiones, la profesión de abogado también parece desaparecer dentro de ese caos.

El Proyecto de Reforma Procesal Civil opta por un sistema legalista que pretende ordenar y sobre todo renovar el caos hoy imperante. Muchas de las actividades académicas a las que hemos podido asistir tratan algunos aspectos concretos de la reforma. Quizá dos son los grandes temas: el sistema de recursos y la ejecución e incluso tres si es que tenemos en cuenta el gran avance que ha de significar el procedimiento monitorio ${ }^{5}$.

En escasas ocasiones, eso sí, hemos podido reflexionar sobre los principios del proceso y del procedimiento. Quizá resulta, para algunos un tema demasiado acadé-

2 Maturana Miquel, Cristian, Reforma Procesal Penal. Génesis, historia sistematizada y concordancias, Ed. Jurídica de las Américas, Santiago, 2009.

3 Montero Aroca, Juan, Derecho Jurisdiccional I. Parte General, Tirant lo Blanch, Valencia, 2011, p. 26.

4 Palomo Vélez, Diego, Las marcas del proceso oral y escrito diseñado en el Proyecto de Nuevo CPC chileno, /en/ Revista Chilena de Derecho, vol. 36, №3, p. 623.

5 Pérez Ragone, Álvaro, En torno al procedimiento monitorio desde el derecho procesal comparado europeo: Caracterización, elementos esenciales y accidentales /en/ Revista de Derecho (Valdivia), vol. 19, N¹, 2006, pp. 205-235. 
mico y distante de la realidad (de la praxis) sin embargo, se nos antoja más cercano de lo que creemos y, en exceso, importante. Cuanto más profundo es el estudio de la Ciencia Procesal más huye de conocimiento de los plazos y más atiende a la importancia de los principios.

Bajo la rúbrica "Principios Básicos" el Libro Primero de las Disposiciones Generales consagra en diez artículos las directrices que han de revolucionar el proceso civil y que consolidan un proceso moderno, actual y que debería resultar eficiente ${ }^{6}$.

De forma bien original se han organizado los principios que la doctrina moderna considera que han de inspirar un debido proceso ${ }^{7}$. En primer lugar, podemos localizar los principios del proceso: El artículo 1 reconoce la contradicción o bilateralidad de la audiencia y el proceso como medio de solución del conflicto. Destaca de modo actual el reconocimiento expreso a la posibilidad de acudir a un árbitro debido al auge que está teniendo en el mundo la solución alternativa de conflictos.

El artículo 2 reconoce el debido proceso. Se entiende este como un principio de legalidad del mismo. La jurisdicción será ejercida por un tribunal que sea competente, imparcial, independiente y que enjuiciará conforme a un procedimiento legalmente establecido ${ }^{8}$.

El artículo 3 establece el principio dispositivo. Famoso es el brocardo latino nemo iudex sine actore. Las partes son dueñas del proceso desde su principiación hasta su eventual conclusión adelantada.

El artículo 4 prescribe una de las más importantes novedades derivadas de la oralidad del procedimiento: el impulso del juez. Ese impulso, entendido como dirección que determina un nuevo rol en los jueces, mucho más activo, más preparado ${ }^{9}$. Los jueces deben adoptar de oficio las medidas oportunas para evitar todo tipo de dilación indebida y conseguir una respuesta judicial lo más rápido posible ${ }^{10}$. Este es

6 Se puede acceder mediante en el siguiente enlace: http://web.minjusticia.cl/ucrpc/estudios/proy-codigo-civil.doc

7 Fernández González, Miguel Ángel, Derecho a la jurisdicción y debido proceso /en/ Revista de Estudios Constitucionales, N¹, 2004, pp. 100 y ss.

8 Muy interesante resulta, en este sentido, Bordalí Salamanca, Andrés, El derecho fundamental a un tribunal independiente e imparcial en el ordenamiento jurídico chileno /en/ Revista de Derecho (Valparaíso), N³3, 2009, pp. 263 a 302.

9 Román Díaz, Fernando, Reflexiones en torno a la propuesta de reforma al procedimiento civil chileno: I. Principios procesales relativos al órgano jurisdiccional /en/ Revista Chilena de Derecho, vol. 34, $\mathrm{N}^{\circ}$ 3, 2007, p. 587.

10 Ortells Ramos, Manuel, Formas del procedimiento y garantías fundamentales del proceso civil /en/ Revista lus et Praxis, Año 16, Nº1, 2010, p. 396. 
uno más de los principios, pero nos parece de vital importancia el nuevo papel que se otorga al juez, especialmente en la primera instancia, es totalmente desconocido hasta el momento y una de las grandes revoluciones ${ }^{11}$.

Finalmente, la igualdad de armas recogida en el artículo 5 del Proyecto: esta se entiende como un mandato al juez quien deberá velar porque se cumpla un equilibrio entre las posibilidades de las partes ${ }^{12}$.

El procedimiento presenta, según estos principios, las siguientes características:

$1^{\circ}$ ORALIDAD. Recogida en el artículo 7, y casi en idénticos términos en el 64, se destaca como el eje principal de esta y de todas las reformas ${ }^{13}$. Todas las actuaciones serán orales y las resoluciones se dictarán en forma verbal. Actualmente, eso sí, hay consenso en que algunas diligencias deben ser todavía escritas y así demanda, reconvención, contestación y recursos fuera de las audiencias seguirán siendo "en papel"14.

$\mathbf{2}^{\circ}$ INMEDIACIÓN. La que será nuestra próxima ley rituaria civil establece que el juez debe decidir conforme a las actuaciones que se practicaron en su presencia. La oralidad es una revolución filosófica del procedimiento, pero la inmediación hace mucho tiempo que hacía falta ${ }^{15}$. El juez que pronuncia la sentencia debe haber asistido a la práctica de las pruebas de donde extrae su convencimiento ${ }^{16}$. Quedando absolutamente prohibida la delegación de funciones confirmado en los artículos 65 y 260.

$3^{\circ}$ CONCENTRACIÓN. Esta previsión del artículo 9 también parece una necesidad moderna insalvable ${ }^{17}$. Los procedimientos deben ser ágiles y apegados a la realidad. La concentración permite ambos objetivos. Vamos a tener una respuesta más rápida y seguramente más cercana a la realidad si es que se practican todas las pruebas (o la gran mayoría) el mismo día. ¿cómo no va a ser mejor reconstruir una historia en un mismo evento que ir contándola por capítulos?

11 Taruffo, Michelle, La prueba de los hechos, Trotta, Madrid, 2005, p. 330.

12 Carnelutti, Francesco, Cómo se hace un proceso, Traducción de Sentís Melendo y Ayerra Medín, Ed. Termis, Bogotá, 2004, p. 164.

13 Palomo Vélez, Diego, Y ahora, tras la experiencia procesal penal ¿la oralidad al proceso civil? Algunas claves y criterios a seguir /en/ lus et Praxis, N², 2004, p. 226.

14 Pérez Ragone, Álvaro y Palomo Vélez, Diego, Oralidad y prueba: comparación y análisis crítico de las experiencias reformadoras del proceso civil en Alemania y España /en/ Revista de Derecho (Valparaíso), N³2, 2009, p. 373.

15 Palomo Vélez, Diego, obr. cit. p. 634.

16 Gómez Orbaneja, Emilio y Herce Quemada, Vicente, Derecho procesal, Madrid, 1949, p. 228

17 Domínguez Balmaceda, Juan Pablo, Reflexiones en torno a la propuesta de reforma al procedimiento civil chileno: III. Principios Procesales Relativos al Procedimiento /en/ Revista Chilena de Derecho, vol. $34, N^{\circ} 3$, p. 596. 
$4^{\circ}$ PUBLICIDAD. El control del órgano jurisdiccional se consigue con la publicidad de su actuar. Es absurdo que tengamos un modelo oral y que lo mantengamos en secreto $^{18}$. Todas las actuaciones serán públicas -como norma general- y de este modo el ciudadano controla a los tribunales de justicia, aunque sabemos que en realidad no hay nadie en esas audiencias.

Además, de estos principios encontramos recogidos de alguna forma en el Proyecto, y así lo ha señalado en alguna ocasión el profesor MATURANA los principios de: Primacía de la aportación de parte sobre la investigación, primacía de la sana crítica y continuidad (una o dos audiencias) ${ }^{19}$.

Hoy en día está fuera de toda duda la ventaja que supone un procedimiento oral, Ilamémosle eminentemente oral, o mixto ${ }^{20}$, si se quiere sobre un procedimiento escrito o preeminentemente escrito ${ }^{21}$. Sin embargo, quizá estemos demonizando en exceso la escritura ignorando las ventajas que nos ha aportado durante este largo tiempo y la necesidad que sigue demostrando en algunas actuaciones, digamos, más reposadas ${ }^{22}$.

Quizá podríamos entender una ferviente declaración a favor de la oralidad en el año 1909 hecha por Chiovenda demandando un cambio sustancial inspirado en los procesos Alemán y Austríaco y su impresionante estadística judicial. Tal vez siguiendo al propio autor podemos convenir que la esencia del propio proceso romano demandaba de una forma eminentemente oral como medio de fomentar la libre convicción del juez ${ }^{23}$, pero desde tiempos más modernos no existe en la doctrina procesal un entusiasmo tan superlativo como el que parece que vivimos con las reformas procesa$\operatorname{les}^{24}$. Quizá debido a que quienes analizan el proceso romano, en seguida, observan cómo se transitó de la oralidad estricta al procedimiento formulario como medio de organizar el proceso.

18 Domínguez Balmaceda, Juan Pablo, obr.cit. p. 598.

19 Maturana Miquel, Cristian, Los principios de la reforma procesal civil [on line], [consultado el 1 de septiembre de 2011]. Disponible en la World Wide Web: www.sofofa.cl/BIBLIOTECA.../12_CMaturana. pdf

20 Véase, Cappelletti, Mauro, Processo orale e processo scritto nel mondo contemporaneo /en/ Giustizia e società, Milan, 1972, p. 151 y ss; Chiovenda, Giuseppe, Instituciones de Derecho procesal civil, vol. III, Madrid, 1940, p. 159; Prieto-Castro Ferrándiz, Leonardo, Oralidad y escritura en el proceso civil, Trabajos y orientaciones prácticas de Derecho Procesal, Madrid, 1964.

21 Cafferata Nores, José, Juicio penal oral, Temas de Derecho Procesal Penal, Depalma, Buenos Aires, 1998 , p. 270.

22 Nieva Fenoll, Jordi, Los problemas de la oralidad /en/ Justicia, No 1-2, 2007, p. 101 y ss.

23 Chiovenda, Giuseppe, obr.cit. p. 154

24 Cappelletti, Mauro, La oralidad y las pruebas en el proceso civil, traducción de Sentís Melendo, EJEA, Buenos Aires, 1972, p. 5 
De modo que después de reconocer las ventajas de que acarrea la reforma y los principios que ella trae consigo, vamos a desvelar algunos de los problemas que puede presentar un abuso de la oralidad.

Un primer supuesto -crítica doctrinalmente clásica- que se puede producir es que la rapidez provoque precipitación y superficialidad en las decisiones de los jueces ${ }^{25}$. Evidentemente, una decisión más pausada, meditada y decididamente más concienzuda pudiendo leer y releer los expedientes podría resultar más acertada en algunos casos. Es una cuestión de calidad de la decisión como diría Taruffo ${ }^{26}$.

Las actuaciones se realizarán en forma oral y tal y como prescriben los artículos 64 y siguientes se tomará convicción de la causa por audiencias que, lamentablemente, imaginamos que no van a ser únicas en el día y por lo tanto los jueces deberán presenciar varias en una misma jornada. Por otra parte, si duran mucho van a provocar la presencia de los jueces pero la ausencia de sus pensamientos deseando haber revisado muchos de los aspectos (por ejemplo: características técnicas de un vehículo) en un informe escrito.

Habitualmente, el problema suscitado en torno a este debate proviene de la creencia que todos los males del proceso derivan de la escritura y todos los principios positivos beben de la mágica fuente de la oralidad (todos, además provienen de la lectura de Rosemberg ${ }^{27}$, Chiovenda y su reafirmación por Berzosa ${ }^{28}$, Montero Aroca y Vázquez Sotelo). En nuestra reforma, la inmediación, la concentración o la publicidad son ventajas y bondades que descienden de la oralidad.

Pero deberíamos observar todo en su contexto: Ya dijimos que la publicidad es ciertamente relativa cuando nadie acude a presenciar esas audiencias, o los que van no entienden nada. La inmediación también es cuestionable cuando los jueces han de ver desfilar ante sí padres o más dramáticamente madres que avalistas de crédito Iloran por las deudas de sus hijos. Es más, cuando un juez tiene seis audiencias en una mañana inevitablemente debería revisar las grabaciones para recordar cuál de todas aquellas mujeres Iloraba por qué crédito. Evidentemente, esta afirmación se hace aun y a riesgo de ser rebatido por aquellos que saben descifrar el llamado lenguaje no verbal, las expresiones faciales u otros indicadores de la verdad. Pero lo

25 Fontán Silva, Eduardo José, Los derechos fundamentales de naturaleza procesal en el orden civil. El panorama interno e internacional de su contenido /en/ La Ley, 2000, p 265.

26 Taruffo, Michelle, Investigación judicial y producción de prueba por las partes /en/ Revista de Derecho (Valdivia), vol. 15, N², 2003, p. 207.

27 Rosemberg, Leo, Tratado de Derecho procesal civil, traducción de Ángela Romera Vera, EJEA, Buenos Aires, 1955, p. 395.

28 Berzosa Francos, María Victoria, Principios inspiradores del futuro proceso civil, Presente y futuro del proceso civil, Ed. Bosch, Barcelona, 1998, p. 36. 
más importante es destacar que cuando el juez recibe los escritos también actúa con inmediación ya que el mismo los lee. Y en la Corte de Apelación nadie cuestiona el trabajo de los relatores.

Por otra parte, debemos entender que concentración no significa absolutamente celeridad. Puede que el asunto sea tan complejo que su concentración signifique unidad, pero no necesariamente brevedad temporal.

De modo que señalaremos en las siguientes líneas algunos problemas derivados de la oralidad, algunas ventajas de la escritura, para finalizar enfatizando dónde resulta más útil la oralidad.

\section{Cuestionamientos a la oralidad}

La doctrina ha convenido que la escritura nace de la apellatio romana y de la necesidad de protocolizar las actuaciones para ser revisadas por un superior jerárqui$\mathrm{CO}^{29}$. En lo sustancial no fue sino hasta las reformas napoleónicas que se comenzó a pensar en la restauración de la oralidad (reflejado en los códigos de procedimiento civil y criminal de 1806 y 1808 respectivamente) sin embargo, la gran mayoría de países europeos y el propio código de procedimiento civil chileno continuó conservando la escritura como principio fundamental ${ }^{30}$.

El principal problema que presentaba y presenta este modelo es que pese a que tenemos constancia desde la Novísima Recopilación de la exigencia del juez en las actuaciones, esta presencia ha brillado por su ausencia ${ }^{31}$.

De este modo, Chiovenda y sus discípulos comenzaron a expandir la necesidad de la oralidad, incluso plasmándose en algunas Constituciones europeas. Pero hasta en esas primeras épocas podemos ver cómo la fascinación por la oralidad tuvo momentos de dificultad como las reformas pro escritura de los años 1909 y 1924 de la Ordenanza Procesal alemana de 1877 (ZPO) $)^{32}$.

29 Estrada, Susana Isabel, De la apellatio romana a la casación iberoamericana /en/ Revista Jurídica, Facultad de Ciencias Jurídicas y Ciencias Sociales de la Universidad de Tucumán, № 34, 2002, p. 56.

30 Prieto-Castro Ferrándiz, Leonardo, Precisiones sobre escritura y oralidad en el Derecho Procesal español, Estudios y Comentarios para la teoría y la práctica procesal civil, Ed. Reus, Madrid, 1950, p. 78.

30 Luengo Millán, Miguel y Rabanak Carvajo, Pedro, Los principios inspiradores del proceso laboral, Cuadernos de Derecho Procesal Laboral, McGraw-Hill, Madrid, 1999, p. 101.

31 Prieto-Castro Ferrándiz, Leonardo, La reforma del procedimiento civil en Alemania /en/ Revista de Derecho Privado, 1931, p. 113. 
En definitiva, cuáles son los principales problemas de la oralidad: En primer lugar, los jueces no solo van a atender un caso. Esto genera que en la práctica el juez no pueda conservar todos los matices de las audiencias que ha ido presenciando, y que cuantas más audiencias desarrolle menor será el grado de atención de las mismas.

Otro de los puntos débiles que afortunadamente no es tan complejo en la práctica es la privacidad de las partes. La publicidad, que es imprescindible si queremos que sirva para algo la oralidad ${ }^{32}$, determina que muchos asuntos puedan ser conocidos por terceros: deudas, impagos... temas que seguramente son más delicados que llamarlos materia civil privada. Ahora bien, se mitifica la publicidad como consecuencia de la oralidad ${ }^{33}$. Hoy en día tenemos ya tenemos más publicidad de la que nos pueda aportar la oralidad. La revisión de las bases de datos e incluso de la página web del Poder Judicial confieren mucha más publicidad de lo que una audiencia, a la que asistirán generalmente los interesados, pueda entregar a personas legas en derecho.

Sin duda es mucho más público y productivo tener a disposición las resoluciones de los órganos jurisdiccionales de modo que todos los operadores jurídicos vayamos buscando los casos que nos interesan, que tener audiencias orales sin público o, simplemente, presenciadas por familiares que no están entendiendo nada al carecer, en la generalidad de los casos, de la más mínima formación jurídica. Un abogado buscando jurisprudencia es un agente censor mucho más inquisitivo que un familiar desconocedor de las mínimas formas de un proceso.

Incluso en esa misma línea podría pensarse en permitir el monitoreo por cámaras web como ocurre en algunos tribunales europeos como el Tribunal de Justicia de la Unión Europea y el Tribunal Europeo de Derechos Humanos.

Qué decir acerca de la mejor oratoria o grandilocuencia de algunos abogados. ¿Hay justicia cuando se gana un caso por las mejores armas del letrado? Claro que esto nos lleva al peligroso mundo de por qué solo algunos abogados alegan hoy las causas ante la Corte. Además, es absurdo porque, al contrario, tampoco sería justa la victoria del que tiene una mejor pluma. Y, en todo caso, como nos señaló en alguna ocasión el profesor Maturana las leyes procesales no son el medio de solucionar la desigualdad ${ }^{34}$.

32 Ramos Méndez, Francisco, Para un proceso civil eficaz, Publicacions Universitat Autònoma de Barcelona, Barcelona, 1998, p. 17.

33 Palomo Vélez, Diego, La oralidad en el proceso civil. El nuevo modelo español, Librotecnia, Santiago, 2008, p. 37.

34 Maturana Miquel, Cristian, obr. cit. 
Pero Ilevándolo un poco más a la práctica y tomando como comparación el escenario planteado en España, en ocasiones es poco práctico. Hay veces en que la audiencia preliminar (Ilamada en la reforma) o audiencia previa (Ilamada en España) las partes solo van a reiterar casi literalmente lo que ya decían en sus escritos.

Sucede en esas audiencias que pese a los 12 numerales que prevé el nuevo artículo 254 del Proyecto, no se va a realizar otra actividad que ratificarse en un escrito. No estando en un proceso laboral no habrá conciliación a toda costa; el juez probablemente no va a admitir hechos nuevos debido a que no manejará en profundidad los escritos y no querrá transformar el litigio, los puntos de prueba serán difícilmente fijables en estos términos y probablemente esperará a que la parte desista de sus excepciones, o bien, suspenderá la audiencia para otro día con más calma poder analizar las mismas. Seguramente, este problema sea enteramente trasladable a la aplicación del modelo en Chile ya que el problema radica en la sobrecarga de asuntos a los jueces.

Ese es otro de los problemas que se potencia con la oralidad. En el procedimiento escrito tendremos una demora excesiva, pero cuando hay una sobrecarga en un modelo oral conducirá a que los jueces adopten decisiones precipitadas motivadas por el cansancio de escuchar sucesivas audiencias sin tiempo para meditar.

Evidentemente, son varios los años que llevamos escuchando interminables odas a las ventajas que trae aparejada la oralidad, por lo que considerábamos oportuno poder remarcar algunos de los inconvenientes con el objetivo de repensarlos y, quizás, de poder llegar a mejorar el sistema. Indudablemente, que cuando se cantan las ventajas de la oralidad no son en vano y en ningún caso estamos proponiendo la mantención de la escrituración como principio del procedimiento.

Pero, veamos algunas de sus ventajas: La primera de las bondades que implica la escritura es en palabras de Vázquez Sotelo ${ }^{35}$ que contribuye a centrar y fijar con claridad el objeto del juicio y permite que el letrado exprese de mejor modo sus peticiones o defensas en tanto que deja más espacio a la reflexión. Es motivo suficiente para que hoy se mantengan los escritos de demanda y contestación. Podremos discutir detalles en la audiencia preliminar, pero el litigio va a quedar extremadamente determinado en los escritos. El artículo 231 del Proyecto así lo entiende y prescribe que la demanda deberá contener los hechos, el derecho en que se funda y los medios de prueba de los que el actor pretende valerse. Igualmente, el artículo 248 contempla las mismas precisiones para el demandado que deberá señalar los hechos, el derecho y los medios de prueba en que pretenda fundar sus defensas y excepciones.

35 Vázquez Sotelo, José Luis, Los principios del proceso civil /en/ Justicia, III-IV, 1993, p. 640. 
En ocasiones, además, como comentamos anteriormente simplifica los trámites cuando, por decirlo de algún modo, no tenemos nada más que agregar. En este caso, nos ahorramos una audiencia carente de sentido en que solo indicamos que nos ratificamos en el escrito, acompañando la prueba que ya anunciamos.

Pero también simplifica la tarea del juez. El órgano jurisdiccional no ha de revisar audio, sino que la mera lectura rápida puede hacerle recordar los detalles necesarios para fundar su decisión.

Como señaló el profesor Nieva Fenoll ${ }^{36}$ en un análisis al procedimiento catalán de los años 1714 a 1835 lo que determinó el mayor descontento con la escritura y la encarnizada guerra contra la misma del siglo XIX fue la excesiva demora derivada de la infinita impugnabilidad de todas las resoluciones interlocutorias. Y quizás es totalmente aplicable al día de hoy: no son los escritos lo que nos molesta, sino la paralización eterna que provocan los incidentes. Si unimos ese estancamiento a la ausencia de la presencia del juez, encontramos que un procedimiento escrito en estos términos es insostenible.

Y pasa por ahí, precisamente, la mayor crítica a los modelos tradicionalmente escritos. En que el máximo destinatario de la prueba nunca está presente y, por lo mismo, las declaraciones de las partes o de testigos nunca pueden generar convicción en el órgano jurisdiccional. Esto nos lleva a una apreciación de la prueba literalmente "a peso" como indicaba el maestro Cappelletti. Pero démonos cuenta, entonces, que lo que se quiere decir al exigir inmediación es realmente que el juez escuche a las partes y a los testigos, sin embargo, en ocasiones podría "escuchar" a un perito por escrito y al leerlo por sí mismo ser inmediato.

Pero sin duda, en este nuevo modelo de escritura propuesto en el Proyecto de Código Procesal Civil encontramos una ventaja extra. En sede de contestación de la demanda, el artículo 249 del Proyecto regresa (contra la más arraigada tradición) al modelo del "quien calla otorga". Sabemos que en derecho hoy, quien calla no dice nada. Sin embargo, el Proyecto prescribe que el silencio, la ambigüedad o las respuestas evasivas podrán entenderse como admisión de hechos o autenticidad de documentos. Y, solo excepcionalmente, el juez podrá considerar no aplicar esta norma. De modo, que se está incentivando -por decirlo muy correctamente- a que el demandado sea activo, conteste, lo haga meditadamente $y$, por lo tanto, se requiere de un escrito que nos ayude a determinar los alcances del objeto litigioso.

36 Nieva Fenoll, Jordi, El proceso jurisdiccional catalán de 1714 a 1835 . Breve reseña histórica /en/ Justicia, I-Il 2005, pp. 57 y ss. 


\section{Ventajas de la escritura}

En especial, es necesaria la escritura tal y como reconoció Cappelletti en la fase de discusión. Es extremadamente importante poder reflexionar a cabalidad cuál es el objeto del litigio. Incluso escritos de dúplica y réplica podrían evitar audiencias preliminares -en casos sencillos- y preparar la entrada a la audiencia de juicio. Quizá se podría evitar suspensiones, con la permisión de estos escritos, derivadas de la problemática que pudieran generar las excepciones. Así también lo entendió Chiovenda quien siendo de los mayores defensores de la oralidad caracterizaba la oralidad a raíz de un procedimiento tributario con un "predominio de la palabra hablada como medio de expresión, atenuado por el uso de escritos de preparación y documentación" ${ }^{37}$.

También consideramos, al contrario de lo que prescribe el proyecto, que podrían resultar muy útiles unas conclusiones a la prueba escritas. Sería más conveniente presentar reflexivamente la actividad probatoria y no de forma limitada en el tiempo, poco reflexiva y, en definitiva, abriendo la puerta a la obscuridad. Resulta hasta el punto que el propio artículo 308 indica en su inciso final que el juez podrá solicitar a las partes que aclaren sus dichos. De modo que el diseño reconoce que este momento es crucial y que pueden producirse problemas por un desarrollo demasiado fugaz.

Debemos eliminar la oralidad en los incidentes. Solo si las partes van a llegar a acuerdo podemos celebrarlo. Ahora bien, es más complicado suspender y buscar un día para celebrar una audiencia en un, por seguro, sobrecargado tribunal. Al contrario de lo que podría parecer, además, puede resultar mucho más útil un intercambio de escritos para resolver algo que es accesorio. Precisamente, la implementación de un modelo tendencialmente rápido debe sortear estos obstáculos. Si bien es cierto, que el Proyecto contempla la inmediata resolución de los mismos, si el juez no ha leído -como previsiblemente sucederá- los escritos de demanda y contestación seguramente, opte por la salida que le permite el 129 que prescribe que se pueda recibir prueba en otro momento fijándose, de este modo, otra audiencia.

Por todos estos motivos, es sin duda de aplaudir -aunque por la rumorología que envuelve al Proyecto parece que va a desaparecer- la sentencia definitiva inmediata en el caso de que se produzca un allanamiento total o, y es lo verdaderamente importante, cuando solo se haya de rendir prueba documental. Cuando el objeto es tan sumamente determinado el demandado sólo puede allanarse u oponerse fundadamente aportando documentos. No es necesario realizar otra audiencia para reafirmar el contenido de diáfanos medios de cumplimiento de la obligación. Por eso es remarcable esta solución técnica que mediante la inmediación de la lectura va a llevar a la rapidez que se persigue.

37 Chiovenda, Giuseppe, obr. cit. P. 161. 


\section{Etapas oportunamente orales}

En especial en la prueba. La fase probatoria ha de ser el momento de crear la convicción en el juez, ha de ser la instancia en que podamos expresar qué sucedió conforme a los medios que acompañamos. Pero por sobre todo es el momento en que el juez explote al máximo la prescripción del artículo 4 y sea el director. El juez debe ser activo, debe preguntar y comenzar a adquirir los elementos que poco a poco van a ir formando su convicción. Es imposible que pensemos en un juez que exclusivamente forma su juicio en la sentencia.

También resulta esencial como recoge el artículo 345 que se haya oralidad en la apelación. El litigio está claro y los argumentos han sido expuestos. Debe permitirse a las partes subrayar los matices que derivan en la anulación del juicio oral. Esta, de todos modos, es una cuestión bien controvertida entre los que creen que ya no es el momento de oír más alegaciones en tanto se concibe la apelación como un recurso de nulidad al estilo de la casación. Y quienes consideran que se podrían practicar algunas diligencias probatorias reproduciendo los audios de las audiencias, criticado por quienes entienden que es totalmente distinto fallar cuando no hay inmediación estrictamente directa.

\section{A modo de conclusión}

Es absurdo intentar en este $u$ otro foro sostener que la oralidad debería erradicarse en los procedimientos, en especial cuando la misma hace que el ciudadano pase de ser un legajo a ser un rostro. Pero también es imposible sostener que todo ha de ser oral y que la escritura no sirve como instrumento del proceso. Todo en su justa medida, hemos tratado de resaltar algunos beneficios de la escritura y algunos de los momentos que podrían ayudar a la celeridad desde el plano de la escritura. Antes de que comience a caminar quizá es un buen momento para realizar este tipo de prevenciones en aras a la consecución de una tutela judicial realmente efectiva. Esa debe ser la auténtica finalidad, en ocasiones nos olvidamos de las sabias palabras de Couture quien señalaba que "en el proceso el tiempo no es oro, es justicia".

Quizá la sapiencia de todo sea llegar al término medio en este caso llegaríamos, inevitablemente, a la misma conclusión que Carnelutti: "hablar y escribir no son medios equivalentes sino más bien complementarios ${ }^{\prime 38}$.

38 Carnelutti, Francesco, Derecho Procesal civil y penal, Biblioteca clásicos del Derecho, México, 1994, p. 100. 


\section{Bibliografía}

Berzosa Francos, María Victoria, Principios inspiradores del futuro proceso civil, Presente y futuro del proceso civil, Ed. Bosch, Barcelona, 1998.

Bordalí Salamanca, Andrés, El derecho fundamental a un tribunal independiente e imparcial en el ordenamiento jurídico chileno /en/ Revista de Derecho (Valparaíso), N³3, 2009.

Cafferata Nores, José, Juicio penal oral, Temas de Derecho Procesal Penal, DePalma, Buenos Aires, 1998.

Cappellettı, Mauro, La oralidad y las pruebas en el proceso civil, traducción de Sentís Melendo, EJEA, Buenos Aires, 1972.

Cappeleteti, Mauro, Processo orale e processo scritto nel mondo contemporaneo /en/ Giustizia e società, Milan, 1972.

Carnelutti, Francesco, Cómo se hace un proceso, Traducción de Sentís Melendo y Ayerra Medín, Ed. Termis, Bogotá, 2004.

Carneluttı, Francesco, Derecho Procesal civil y penal, Biblioteca clásicos del Derecho, México, 1994.

Chiovenda, Giuseppe, Instituciones de Derecho procesal civil, vol. III, Madrid, 1940.

Domínguez Balmaceda, Juan Pablo, Reflexiones en torno a la propuesta de reforma al procedimiento civil chileno: III. Principios Procesales Relativos al Procedimiento /en/ Revista Chilena de Derecho, vol. 34, Nº 3.

EstradA, Susana Isabel, De la apellatio romana a la casación iberoamericana /en/ Revista Jurídica, Facultad de Ciencias Jurídicas y Ciencias Sociales de la Universidad de Tucumán, No 34, 2002.

Fernández González, Miguel Ángel, Derecho a la jurisdicción y debido proceso /en/ Revista de Estudios Constitucionales, № 1, 2004.

Fontán SiLVA, Eduardo José, Los derechos fundamentales de naturaleza procesal en el orden civil. El panorama interno e internacional de su contenido /en/ La Ley, 2000.

Gómez Orbaneja, Emilio y Herce Quemada, Vicente, Derecho procesal, Madrid, 1949.

Luengo Millán, Miguel y Rabanak Carvajo, Pedro, Los principios inspiradores del proceso laboral, Cuadernos de Derecho Procesal Laboral, McGraw-Hill, Madrid, 1999.

Maturana Miquel, Cristian, Los principios de la reforma procesal civil [on line], [consultado el 1 de septiembre de 2011]. Disponible en la World Wide Web: www. sofofa.cl/BIBLIOTECA.../12_CMaturana.pdf

Maturana Miquel, Cristian, Reforma Procesal Penal. Génesis, historia sistematizada y concordancias, Ed. Jurídica de las Américas, Santiago, 2009. 
Montero Aroca, Juan, Derecho Jurisdiccional I. Parte General, Tirant lo Blanch, Valencia, 2011.

Nieva Fenoll, Jordi, El proceso jurisdiccional catalán de 1714 a 1835 . Breve reseña histórica /en/ Justicia, I-II 2005.

Nieva Fenoll, Jordi, Los problemas de la oralidad /en/ Justicia, № 1-2, 2007.

Ortells Ramos, Manuel, Formas del procedimiento y garantías fundamentales del proceso civil /en/ Revista lus et Praxis, Año 16, N 1, 2010.

Palomo Vélez, Diego, La oralidad en el proceso civil. El nuevo modelo español, Librotecnia, Santiago, 2008.

Palomo Vélez, Diego, Las marcas del proceso oral y escrito diseñado en el Proyecto de Nuevo CPC chileno, /en/ Revista Chilena de Derecho, vol. 36, Nº 3.

Palomo Vélez, Diego, Y ahora, tras la experiencia procesal penal ¿la oralidad al proceso civil? Algunas claves y criterios a seguir /en/ lus et Praxis, № 2, 2004.

Pérez Ragone, Álvaro y Palomo Vélez, Diego, Oralidad y prueba: comparación y análisis crítico de las experiencias reformadoras del proceso civil en Alemania y España /en/ Revista de Derecho (Valparaíso), N³ 32, 2009.

Pérez Ragone, Álvaro, En torno al procedimiento monitorio desde el derecho procesal comparado europeo: Caracterización, elementos esenciales y accidentales /en/ Revista de Derecho (Valdivia), vol. 19, № 1, 2006.

Prieto-Castro Ferrándiz, Leonardo, La reforma del procedimiento civil en Alemania / en/ Revista de Derecho Privado, 1931.

Prieto-Castro Ferrándiz, Leonardo, Precisiones sobre escritura y oralidad en el Derecho Procesal español, Estudios y Comentarios para la teoría y la práctica procesal civil, Ed. Reus, Madrid, 1950.

Prieto-Castro Ferrándiz, Leonardo, Oralidad y escritura en el proceso civil, Trabajos y orientaciones prácticas de Derecho Procesal, Madrid, 1964.

Ramos Méndez, Francisco, Para un proceso civil eficaz, Publicacions Universitat Autònoma de Barcelona, Barcelona, 1998.

Román Díaz, Fernando, Reflexiones en torno a la propuesta de reforma al procedimiento civil chileno: I. Principios procesales relativos al órgano jurisdiccional / en/ Revista Chilena de Derecho, vol. 34, N 3, 2007.

RosemberG, Leo, Tratado de Derecho procesal civil, traducción de Ángela Romera Vera, EJEA, Buenos Aires, 1955.

TARUFFO, Michelle, Investigación judicial y producción de prueba por las partes /en/ Revista de Derecho (Valdivia), vol. 15, N² 2003.

TARuffo, Michelle, La prueba de los hechos, Trotta, Madrid, 2005.

VÁzquez Sotelo, José Luis, Los principios del proceso civil /en/ Justicia, III-IV, 1993. 